\title{
Microlensing Diagnosis in Lensed Quasars
}

\author{
V. Motta ${ }^{1}$ E. Mediavilla ${ }^{2}$, E. E. Falco ${ }^{3}$ and J. A. Muñoz ${ }^{4}$ \\ ${ }^{1}$ Departamento de Fisica y Astronomia, Universidad de Valparaiso, Avda. Gran Bretaña 1111, \\ Valparaiso, Chile \\ email: vmotta@dfa.uv.cl \\ ${ }^{2}$ Instituto de Astrofisica de Canarias, Universidad de La Laguna, V'ia Láctea s/n, La Laguna, \\ Tenerife, España \\ email: emg@iac.es \\ ${ }^{3}$ Harvard-Smithsonian Center for Astrophysics, Whipple Observatory, Amado, Arizona, USA \\ email: falco@cfa.harvard.edu \\ ${ }^{4}$ Departament d'Astronomia i Astrofisica, Universitat de València, Burjassot, Valencia, España \\ email: jmunoz@uv.es
}

Simple gravitational lens models usually suffice to reproduce the positions of lensed quasar images, but they have problems to reproduce their optical flux ratios. The socalled flux ratio anomalies are thought to be produced by small-scale structure in the lens galaxies (microlensing).

Since the sizes of the emitting regions in each quasar depend on the observed wavelength, microlensing will yield a wavelength-dependent magnification: the continuum emitting region is magnified but not the narrow-line region. For a pair of images, the ratio of their emission line fluxes represents the baseline of no microlensing. Relative to this, the ratio of their continua yields the difference in microlensing magnification. We demonstrate the method, presenting the results obtained from MMT, VLT and HST spectroscopy for Q0957+561 and HE1104-1805. In these cases we found the microlensing magnification is wavelength-dependent (chromatic). A statistically significant sample of microlensing measurements using spectra will allow us to estimate the fraction of mass in the lens galaxy that is composed of compact objects $(\alpha)$.

We conducted a preliminary study, with microlensing measurements from spectroscopy we collected from the literature for 29 image pairs. The histogram of observed microlensing events peaks below $0.6 \mathrm{mag}$. The likelihood of the microlensing measurements using frequency distributions obtained from simulated microlensing magnification maps is explained by a low value of $\alpha(<10 \%)$. The results will improve once a homogenous and statistically significant sample of microlensing measurements is assembled. 